CALT-68-1903

hep-ph/9311369

DOE RESEARCH AND

DEVELOPMENT REPORT

\title{
Evaluation of the One-loop Effective Action at Zero and Finite Temperature*
}

\author{
Clarence L. Y. Lee \\ California Institute of Technology, Pasadena, CA 91125
}

\begin{abstract}
A method for determining the leading quantum contributions to the effective action for both zero and finite temperatures is presented. While it is described in the context of a scalar field theory, it can be straight-forwardly extended to include fermions. An extrapolation procedure which can significantly enhance the computational efficiency is introduced. This formalism is used to investigate quantum corrections to the nucleation rate in first-order phase transitions.
\end{abstract}

October, 1993

*Work supported in part by the U.S. Dept. of Energy under Contract No. DE-FG03-92-ER40701. 


\section{Introduction}

The evaluation of quantum corrections to classical solutions is an important problem which pervades much of modern theoretical physics. However, while effective potentials have been studied extensively, methods for determining the effective action are less well-developed. Moreover, the actual evaluation of such effects for realistic systems has often been hampered by their general intractability to analytical solution and the lack of efficient computational methods. ${ }^{[1-5]}$

In the effective potential approximation to the effective action, quantum fluctuations are integrated out about a constant classical field - but this is not expected to be adequate because the classical field is generally an inhomogeneous configuration. The derivative ex-

pansion $^{[6]}$ improves on this by accounting for spatially varying background fields; its leading term is the effective potential. The expansion is a perturbative approximation which extracts the dominant contribution of short-distance quantum effects on long-distance physics. When it converges, it provides an efficient means for performing calculations. However, when it diverges, one must often resort to brute-force techniques which entail an explicit, computationally-intensive evaluation. Furthermore, the derivative expansion fails whenever the potential $V$ is non-convex $\left(V^{\prime \prime}<0\right)$ in some region of space, which includes an important class of perturbatively calculated potentials. ${ }^{[7]}$ It is clear that a general method, which is also applicable to such cases, is needed.

In this paper, a method for calculating the quantum effects arising from the effective action is presented. The next section contains the general formalism for evaluating the effective action. Section 3 discusses the exact formulation of the computational method as well as extrapolation techniques which improve its convergence properties. This formalism is applied to the analysis of phase transitions in Section 4. ${ }^{[7,8]}$

\section{General Formalism}

Consider a scalar field theory with the Lagrangian density

$$
\mathcal{L}(\phi)=\frac{1}{2} \partial_{\mu} \phi \partial^{\mu} \phi-V(\phi)
$$

where $V$ is the tree-level potential which has a (classical) vacuum at $\phi_{v}$. Since this paper considers $3+1$ dimensions exclusively, renormalizability constrains $V$ to have no polynomials in $\phi$ of higher power than a quartic. The classical field $\tilde{\phi}$ is determined by the equation

$$
\partial_{\mu} \partial^{\mu} \tilde{\phi}=-V^{\prime}(\tilde{\phi})
$$


The contribution of one-loop quantum effects to the effective action can be written as

$$
\operatorname{Tr} \mathcal{L}^{(1)}=\frac{i}{2} \operatorname{Tr} \ln \left|\frac{\partial^{2}+V^{\prime \prime}(\tilde{\phi})}{\partial^{2}+\mu^{2}}\right|
$$

where $\mu^{2}=V^{\prime \prime}\left(\phi_{v}\right)$ and the trace runs over space-time coordinates. ${ }^{\dagger}$ Part of this trace can be evaluated as $\operatorname{Tr} \mathcal{L}^{(1)}=\operatorname{Tr}^{\prime} \int\left\langle t\left|\mathcal{L}^{(1)}\right| t\right\rangle d t$, where $\operatorname{Tr}^{\prime}$ runs over the remaining spatial degrees of freedom. Since this paper deals only with time-independent $\tilde{\phi}$ fields, specializing to this case means that states in the energy basis $|\omega\rangle$ are eigenstates of the operator in $\mathcal{L}^{(1)}$. So inserting a complete set of such states and performing a partial integration yields

$$
\operatorname{Tr} \mathcal{L}^{(1)}=-i \int \operatorname{Tr}^{\prime} \int\left[\frac{1}{-\omega^{2}-\nabla^{2}+V^{\prime \prime}(\tilde{\phi})}-\frac{1}{-\omega^{2}-\nabla^{2}+\mu^{2}}\right] \omega^{2} \frac{d \omega}{2 \pi} d t .
$$

Observe the non-locality of this expression; this generic feature of loop corrections makes exact analytical treatments difficult. The remaining trace can be conveniently performed over the eigenstates of the operators in $\mathcal{L}^{(1)}$ : if $\psi_{j}^{0}$ and $\psi_{j}$ are chosen such that

$$
\begin{gathered}
{\left[-\nabla^{2}+\mu^{2}\right] \psi_{j}^{0}=\left(\omega_{j}^{0}\right)^{2} \psi_{j}^{0},} \\
{\left[-\nabla^{2}+V^{\prime \prime}(\tilde{\phi})\right] \psi_{j}=\left(\omega_{j}\right)^{2} \psi_{j},}
\end{gathered}
$$

where the subscript $j$ indexes the eigenstates, then

$$
\operatorname{Tr} \mathcal{L}^{(1)}=-\frac{1}{2} \int \sum_{j}\left(\omega_{j}-\omega_{j}^{0}\right) d t
$$

Hence the one-loop effective action can be written as

$$
S_{\text {eff }}(\tilde{\phi})=\int\left[\mathcal{L}(\tilde{\phi})-\mathcal{L}\left(\phi_{v}\right)\right] d^{4} x+\int\left[-\frac{1}{2} \sum_{\omega_{j}^{0}<\Lambda}\left(\omega_{j}-\omega_{j}^{0}\right)+\int \mathcal{L}_{\mathrm{ct}}(\tilde{\phi}, \Lambda) d^{3} x\right] d t
$$

The bare sum in $\operatorname{Tr} \mathcal{L}^{(1)}$ is divergent; it is regulated in eq. (2.5) by a momentum cut-off $\Lambda$, and a counterterm $\mathcal{L}_{\mathrm{ct}}(\tilde{\phi}, \Lambda)$ has been added to render it finite.

For time-independent fields $\tilde{\phi}$, it is more convenient to focus on the energy $E$ of the system which is related to $S_{\text {eff }}$ through

$$
S_{\mathrm{eff}}(\tilde{\phi})=-E(\tilde{\phi}) \int d t
$$

${ }^{\dagger}$ The trace excludes possible negative and zero modes of the operator $\partial^{2}+V^{\prime \prime}(\tilde{\phi})$. When such modes arise, they must be explicitly removed and treated differently. ${ }^{[7]}$ 
Then

$$
E(\tilde{\phi})=E_{\mathrm{cl}}(\tilde{\phi})+E^{(1)}(\tilde{\phi}, \Lambda)+E_{\mathrm{ct}}(\tilde{\phi}, \Lambda),
$$

where $E_{\mathrm{cl}}$ is the energy of the classical field configuration

$$
E_{\mathrm{cl}}(\tilde{\phi})=-\int\left[\mathcal{L}(\tilde{\phi})-\mathcal{L}\left(\phi_{v}\right)\right] d^{3} x
$$

$E^{(1)}$ is the one-loop contribution

$$
E^{(1)}(\tilde{\phi}, \Lambda)=\frac{1}{2} \sum_{\omega_{j}^{0}<\Lambda}\left(\omega_{j}-\omega_{j}^{0}\right),
$$

and $E_{\mathrm{ct}}$ is the energy due to the one-loop counterterms

$$
E_{\mathrm{ct}}(\tilde{\phi}, \Lambda)=-\int \mathcal{L}_{\mathrm{ct}}(\tilde{\phi}, \Lambda) d^{3} x
$$

At finite temperature $T$, the free energy $F$ replaces $E:[7]$

$$
F_{\mathrm{cl}}=E_{\mathrm{cl}}, \quad \Delta F_{1}=E^{(1)}, \quad F^{\mathrm{ct}}=E_{\mathrm{ct}} ;
$$

for bosons, $\tilde{\phi}(\vec{x}, \tau)$ is periodic in Euclidean time $\tau$ with period $T^{-1}$, and there is an additional contribution due to one-loop effects: ${ }^{[7]}$

$$
\Delta F_{T}=T \sum_{j} \ln \left(\frac{1-e^{-\omega_{j} / T}}{1-e^{-\omega_{j}^{0} / T}}\right)
$$

Observe that no additional counterterms need to be added to $F^{\text {ct }}$ because finite temperatures do not change the short-distance behaviour of the theory.

In the following section, we describe the method developed to evaluate the the quantum corrections, $\Delta F_{1}$ and $\Delta F_{T}$, formally given by eq. (2.8) and (2.11). While for some special situations, the $\omega_{j}$ can be obtained analytically, this is unfortunately not possible for a general potential $V^{\prime \prime}(\tilde{\phi})$. Instead the eigenvalues must be found numerically, then for $\Delta F_{1}$, the bare sum $\sum_{\omega_{j}^{0}<\Lambda}\left(\omega_{j}-\omega_{j}^{0}\right)$ is computed explicitly, and finally the counterterm subtracted; for $\Delta F_{T}$, the sum in eq. (2.11) must be performed term-by-term. To attain reasonable accuracy this subtraction has to be done at a large cut-off $\Lambda$ (to achieve convergence) when both the bare sum and the counterterm (which individually diverge as a function of the cut-off) are numerically very large. Since the final result is much smaller, each term has to be determined very precisely, resulting in a heavy computational burden. Furthermore, the straight-forward approach of evaluating the free energy by a "brute-force" term-by-term summation of the expressions in eq. (2.8) and (2.11) until convergence is reached is also computationally inefficient. 


\section{Method of Computation}

To circumvent the above-described problem of having to compute both the regulated bare sum and its counterterm to very high numerical accuracy, the three-dimensional problem is first decomposed into channels of definite angular momentum. Then for each channel, the divergent part of the bare sum is analytically removed through subtraction with the corresponding divergence in the counterterm, leaving a much smaller finite piece. Since the contribution of higher partial-wave channels decrease rapidly, this procedure overcomes the problem.

An improved computational method is then presented. It is based in part on the observation that the higher-energy modes in the spectrum of eq. (2.4) are less perturbed by the potential $V^{\prime \prime}(\tilde{\phi})$ due to the non-uniform background field $\tilde{\phi}$ than the lower-energy ones. This allows us to formulate an approximation method which accounts for the contribution of the high-energy modes accurately (where the accuracy of the approximation increases with the energy) so that only some of the lower-energy modes need to be treated exactly. ${ }^{[2]}$

\section{Exact Formulation}

The difference in the eigenenergies $\omega_{j}$ and $\omega_{j}^{0}$ of the unbound states $(\omega>\mu)$ can be characterized by the phase shift between the (asymptotic forms) of the corresponding continuum state eigenfunctions $\psi_{j}$ and $\psi_{j}^{0}$, as was first shown in one dimension. ${ }^{[1]}$ Since the phase shift is generally a well-behaved, smoothly-varying function of the energy, it is relatively easy to calculate. Hence it is convenient to express the free energy in terms of this quantity. To determine the phase shift, we consider eq. (2.4) which determines the fluctuations about the classical field configuration.

Since most classical solutions $\tilde{\phi}$ exhibit spherical symmetry $(\tilde{\phi}=\tilde{\phi}(r))$, we will restrict our analysis to such systems. Then the solution to eq. (2.4) can be separated into radial and angular parts by choosing an eigenfunction of the form

$$
\psi_{n l m}(r, \theta, \phi)=\frac{1}{r} u_{n l}(r) Y_{l m}(\theta, \phi),
$$

where the radial wavefunction is determined by

$$
\left[-\frac{d^{2}}{d r^{2}}+\frac{l(l+1)}{r^{2}}+V^{\prime \prime}(\tilde{\phi}(r))-\omega_{n, l}^{2}\right] u_{n, l}(r)=0
$$

with the boundary condition $u_{n, l}(0)=0$. The $Y_{l m}$ are the spherical harmonics corresponding to a state with total angular momentum $l$ and $z$-component $m$.

The corresponding equation for $u_{n, l}^{0}$ where $V^{\prime \prime}(\tilde{\phi})$ is replaced by $\mu^{2}$,

$$
\left[-\frac{d^{2}}{d r^{2}}+\frac{l(l+1)}{r^{2}}+\mu^{2}-\left(\omega_{n, l}^{0}\right)^{2}\right] u_{n, l}^{0}(r)=0,
$$


has an exact analytical solution:

$$
u_{n l}^{0}(r)=\sqrt{2} k_{n} r j_{l}\left(k_{n} r\right)
$$

where $j_{l}$ is a spherical Bessel function and $k_{n}^{2}=\omega_{n}^{2}-\mu^{2}$. These solutions have the asymptotic form

$$
u_{n l}^{0}(r) \rightarrow \sqrt{2} \sin \left(k_{n} r-\frac{l \pi}{2}\right), r \rightarrow \infty
$$

The potentials we consider behave asymptotically as $V^{\prime \prime}(\tilde{\phi}(r)) \rightarrow \mu^{2}$ when $r \rightarrow \infty$ (which corresponds to those with finite action). For such potentials, the asymptotic behaviour of the solution to eq. (3.2) will be

$$
u_{n l}(r) \rightarrow \sqrt{2} \sin \left(k_{n} r-\frac{l \pi}{2}+\delta_{l}\left(k_{n}\right)\right), r \rightarrow \infty
$$

These equations serve to define the phase shift $\delta_{l}$ for each angular momentum channel $l$. Note also that both $u_{n, l}$ and $u_{n, l}^{0}$ are $(2 l+1)$-fold degenerate.

To facilitate the counting of states, it is convenient to discretize the eigenvalue spectrum. This procedure can be achieved by enclosing the system in a box of radius $L$ (where $L$ is much greater than the range of the potential $V^{\prime \prime}$ ) and imposing the boundary condition

$$
u_{n, l}(L)=0
$$

which requires that

$$
k_{n} L-\frac{l \pi}{2}+\delta_{l}\left(k_{n}\right)=n \pi .
$$

Note that such a discretization is implicit in the formal sums in eq. (2.8) and (2.11). The values attained by $\omega^{0}$ (before discretization) as defined by the energy eigenvalue of eq. (3.3) is a continuous spectrum ranging from an energy of $\mu$ to infinity. The corresponding spectrum for $\omega$ determined by eq. (3.2) will generally consist of some discrete bound states with energies $\omega_{j}^{2}<\mu^{2}$ and a continuous spectrum with energies $\omega_{j}^{2}>\mu^{2}$. The difference in structure between the continuum spectra of the two systems manifests in a difference in the respective density of states. Hence it is appropriate to express the sum over eigenenergies for the states in the continuum as an integral over the density of states:

$$
\begin{gathered}
\sum_{j} \omega_{j}^{0}=\sum_{l}(2 l+1) \int_{\mu}^{\infty} \omega n_{l}^{0}(\omega) d \omega \\
\sum_{j} \omega_{j}=\sum_{\omega_{n l}^{2}<\mu^{2}}(2 l+1) \omega_{n l}+\sum_{l}(2 l+1) \int_{\mu}^{\infty} \omega n_{l}(\omega) d \omega
\end{gathered}
$$


where $(2 l+1) n_{l}(\omega)$ is the density of states of angular momentum $l$ for the potential $V^{\prime \prime}(\tilde{\phi})$ with an analogous definition for $n_{l}^{0}$. On taking the continuum limit $(L \rightarrow \infty)$, it follows from eq. (3.8) that the densities of states are related to the continuum phase shift through

$$
n_{l}(\omega)=n_{l}^{0}(\omega)+\frac{1}{\pi} \frac{d \delta_{l}(\omega)}{d \omega}
$$

Now if eq. (2.4) has $N$ bound states, then since eq. (2.4) and eq. (2.3) must have the same total number of states,

$$
N+\sum_{l}(2 l+1) \int_{\mu}^{\infty} n_{l}(\omega) d \omega=\sum_{l}(2 l+1) \int_{\mu}^{\infty} n_{l}^{0}(\omega) d \omega .
$$

For a finite potential, this implies $N \pi=\delta(\mu)$.

It is convenient to define the free energy in each angular momentum channel such that

$$
\begin{aligned}
\Delta F_{1}(\Lambda) & =\sum_{l}(2 l+1) \Delta F_{1}^{l}(\Lambda), \\
\Delta F_{T} & =\sum_{l}(2 l+1) \Delta F_{T}^{l},
\end{aligned}
$$

and to similarly partition the counterterm energy as

$$
F^{\mathrm{ct}}(\Lambda)=\sum_{l}(2 l+1) F_{l}^{\mathrm{ct}}(\Lambda)
$$

then from the above equations

$$
\Delta F_{1}^{l}(\Lambda)=\frac{1}{2} \sum_{\omega_{n l}^{2}<\mu^{2}}\left(\omega_{n l}-\mu\right)-\frac{1}{2 \pi} \sum_{l} \int_{\mu}^{\Lambda} \delta_{l}(\omega) d \omega
$$

and

$$
\Delta F_{T}^{l}=T \sum_{\omega_{n l}^{2}<\mu^{2}} \ln \left(\frac{1-e^{-\omega_{n l} / T}}{1-e^{-\mu / T}}\right)-\frac{1}{\pi} \int_{\mu}^{\infty} \frac{\delta_{l}(\omega)}{e^{\omega / T}-1} d \omega .
$$

The Appendix discusses the renormalization of the Lagrangian given by eq. (2.1). It is shown there that the contribution of the counterterms to the energy are of the general form

$$
\int\left[g\left(\omega^{2}-p^{2}-\mu^{2}\right) \int h(x) d^{3} x\right] \frac{d^{3} p}{(2 \pi)^{3}} \frac{d \omega}{2 \pi} \equiv \int_{-\infty}^{\infty} \operatorname{Tr}^{\prime}(g h) \frac{d \omega}{2 \pi},
$$

where $g$ is a power of the propagator, $h$ is a function of $\phi$ and its derivatives, and $\operatorname{Tr}^{\prime}$ is a trace over the spatial variables. The partial wave decomposition of these contributions 
is achieved by taking the trace with respect to the eigenstates of eq. (2.3) denoted here by $|n l m\rangle$ :

$$
\begin{aligned}
\operatorname{Tr}^{\prime}(g h) & =\sum_{n l m} \sum_{n^{\prime} l^{\prime} m^{\prime}}\left\langle n l m\left|g\left(\omega^{2}+\nabla^{2}-\mu^{2}\right)\right| n^{\prime} l^{\prime} m^{\prime}\right\rangle\left\langle n^{\prime} l^{\prime} m^{\prime}|h(r)| n l m\right\rangle \\
& =\frac{1}{\pi} \sum_{l}(2 l+1) \int_{0}^{\infty}\left[g\left(\omega^{2}-p^{2}-\mu^{2}\right) \int_{0}^{\infty} h(r)\left|u_{p l}^{0}(r)\right|^{2} d r\right] d p .
\end{aligned}
$$

From the Appendix, the counterterm contribution to the free energy is

$$
F^{\mathrm{ct}}(\Lambda)=-\frac{i}{2} \int_{-\infty}^{\infty} \operatorname{Tr}^{\prime}\left\{\Delta_{0}(\omega, p)\left[m^{2}(r)-\mu^{2}\right]+\frac{1}{2} \Delta_{0}(\omega, p)^{2}\left[m^{2}(r)-\mu^{2}\right]^{2}\right\} \frac{d \omega}{2 \pi}
$$

where

$$
\Delta_{0}(\omega, p)=\frac{1}{\omega^{2}-p^{2}-\mu^{2}+i \epsilon}
$$

and $m^{2}(r)=V^{\prime \prime}(\tilde{\phi}(r))$. Evaluating the trace using eq. (3.19) yields

$$
\begin{aligned}
F_{l}^{\mathrm{ct}}(\Lambda)=\int_{0}^{\Lambda_{p}}\{ & -\frac{1}{4 \pi} \frac{1}{\left(p^{2}+\mu^{2}\right)^{1 / 2}} \int_{0}^{\infty}\left|u_{p l}^{0}(r)\right|^{2}\left[m^{2}(r)-\mu^{2}\right] d r \\
& \left.+\frac{1}{16 \pi} \frac{1}{\left(p^{2}+\mu^{2}\right)^{3 / 2}} \int_{0}^{\infty}\left|u_{p l}^{0}(r)\right|^{2}\left[m^{2}(r)-\mu^{2}\right]^{2} d r\right\} d p
\end{aligned}
$$

where $\Lambda_{p}=\sqrt{\Lambda^{2}-\mu^{2}}$ is a three-momentum cut-off.

This completes the formulation of the method for the exact calculation of the free energy. However, as we have remarked above, the convergence of such an exact computation can be sufficiently slow so that extrapolation techniques can be useful. Amongst the various such procedures, we consider in particular the WKB approximation, which provides an analytic expression for the phase shift that is valid at high energies and hence can significantly reduce the effort required to evaluate the phase shift integral. ${ }^{[2]}$

\section{WKB-Improved Method}

A differential equation of the form

$$
\left[\frac{d^{2}}{d x^{2}}+k^{2}(x)\right] f(x)=0
$$

has an approximate WKB solution given by

$$
f_{W K B}(x)=\frac{\exp \left[i \int_{0}^{x} k(\omega, y) d y\right]}{\sqrt{k(\omega, x)}}
$$


which is valid when the wavelength is much less than the distance scale over which $k$ varies:

$$
\frac{1}{k^{2}} \frac{d k}{d x} \ll 1
$$

where $k(\omega, x)$ is the local wavenumber

$$
k(\omega, x)=\sqrt{\omega^{2}-V^{\prime \prime}(\tilde{\phi}(x))} .
$$

Hence the accuracy of the WKB approximation increases with energy. The phase shift for such solutions is given by

$$
\delta^{\mathrm{WKB}}(\omega)=\int_{-\infty}^{\infty}\left[k(\omega, x)-\lim _{y \rightarrow \infty} k(\omega, y)\right] d x
$$

Explicitly,

$$
\delta_{l}^{\mathrm{WKB}}(\omega)=\int_{a(\omega)}^{\infty} \sqrt{\omega^{2}-m^{2}(r)-\frac{l(l+1)}{r^{2}}} d r-\int_{a_{0}(\omega)}^{\infty} \sqrt{\omega^{2}-\mu^{2}-\frac{l(l+1)}{r^{2}}} d r
$$

where $a$ and $a_{0}$ denote the classical turning points defined by

$$
\omega^{2}-m^{2}(a)-\frac{l(l+1)}{a^{2}}=0, \quad \text { and } \quad \omega^{2}-\mu^{2}-\frac{l(l+1)}{a_{0}^{2}}=0 .
$$

Applying this method to eq. (3.2) yields an analytic expression for the energy integral of the phase shift:

$$
\begin{aligned}
\int_{\mu}^{\Lambda} \delta_{l}^{W K B}(\omega) d \omega=\int_{0}^{\infty} & {\left[\int_{\Omega(r)}^{\Lambda} \sqrt{\omega^{2}-m^{2}(r)-\frac{l(l+1)}{r^{2}}} \theta(\Lambda-\Omega(r)) d \omega\right.} \\
& \left.-\int_{\Omega_{0}(r)}^{\Lambda} \sqrt{\omega^{2}-\mu^{2}-\frac{l(l+1)}{r^{2}}} \theta\left(\Lambda-\Omega_{0}(r)\right) d \omega\right] d r
\end{aligned}
$$

with

$$
\Omega(r)=\sqrt{m^{2}(r)+\frac{l(l+1)}{r^{2}}}, \quad \Omega_{0}(r)=\sqrt{\mu^{2}+\frac{l(l+1)}{r^{2}}},
$$

and $\theta(x)$ is the unit step-function. Observe that since the high-energy behaviour of the phase shift is independent of the angular momentum, the energy of each angular momentum channel is logarithmically divergent:

$$
\Delta F_{1}^{l}(\Lambda)=\frac{1}{4 \pi} \ln \left(\frac{\Lambda}{\mu}\right) \int_{0}^{\infty}\left[m^{2}(r)-\mu^{2}\right] d r+\mathcal{O}\left(\Lambda^{0}\right)
$$

Now the divergent piece in $\Delta F_{1}^{l}$ can be analytically combined with the infinite part of $F_{l}^{\text {ct }}$ in eq. (3.21) to leave only finite terms. Performing this subtraction and taking the limit $\Lambda \rightarrow \infty$ 
gives the final expression for the WKB-improved, temperature-independent renormalized free energy:

$$
\begin{aligned}
\Delta F_{1, \mathrm{ren}}^{l}= & \lim _{\Lambda \rightarrow \infty}\left[\Delta F_{1}^{l}(\Lambda)+F_{l}^{\mathrm{ct}}(\Lambda)\right] \\
= & \frac{1}{2} \sum_{\omega_{n l}^{2}<\mu^{2}}\left(\omega_{n l}-\mu\right)-\frac{1}{2 \pi} \int_{\mu}^{\Lambda_{\mathrm{WKB}}} \delta_{l}(\omega) d \omega \\
& -\frac{1}{2 \pi} \int_{0}^{\infty}\left\{\chi_{l}\left(\Lambda_{\mathrm{WKB}}, r\right)+\kappa_{l}(r)\left[m^{2}(r)-\mu^{2}\right]+\rho_{l}(r)\left[m^{2}(r)-\mu^{2}\right]^{2}\right\} d r
\end{aligned}
$$

In this equation, $\chi_{l}$ is the contribution from the WKB phase shift above $\Lambda_{\mathrm{WKB}}$,

$$
\begin{aligned}
\chi_{l}(r)= & \frac{m_{l}^{0}(r)^{2}-m_{l}(r)^{2}-\frac{1}{2} \Lambda_{\mathrm{eff}}(r) \sqrt{\Lambda_{\mathrm{eff}}(r)^{2}-m_{l}(r)^{2}}}{4} \\
& +\frac{1}{2} \Lambda_{\mathrm{eff}}^{0}(r) \sqrt{\Lambda_{\mathrm{eff}}^{0}(r)^{2}-m_{l}^{0}(r)^{2}} \\
& +\frac{1}{2} m_{l}(r)^{2} \ln \frac{\Lambda_{\mathrm{eff}}(r)+\sqrt{\Lambda_{\mathrm{eff}}(r)^{2}-m_{l}(r)^{2}}}{\mu} \\
& -\frac{1}{2} m_{l}^{0}(r)^{2} \ln \frac{\Lambda_{\mathrm{eff}}^{0}(r)+\sqrt{\Lambda_{\mathrm{eff}}^{0}(r)^{2}-m_{l}^{0}(r)^{2}}}{\mu},
\end{aligned}
$$

where

$$
\begin{gathered}
m_{l}^{0}(r)^{2}=\mu^{2}+\frac{l(l+1)}{r^{2}}, \quad m_{l}(r)^{2}=m^{2}(r)+\frac{l(l+1)}{r^{2}}, \\
\Lambda_{\mathrm{eff}}^{0}(r)=\max \left(\Lambda_{\mathrm{WKB}}, \Omega_{0}(r)\right), \quad \Lambda_{\mathrm{eff}}(r)=\max \left(\Lambda_{\mathrm{WKB}}, \Omega(r)\right),
\end{gathered}
$$

and $\Lambda_{\mathrm{WKB}}$ denotes the energy above which the phase shift is computed by the WKB method. The remaining terms in the last integral come from finite parts of the counterterm with

$$
\kappa_{l}(r)=\int_{0}^{\infty} \frac{s^{2}\left|j_{l}(s)\right|^{2}-\frac{1}{2}}{\sqrt{s^{2}+(\mu r)^{2}}} d s,
$$

and

$$
\rho_{l}(r)=-\frac{r^{2}}{4} \int_{0}^{\infty} \frac{s^{2}\left|j_{l}(s)\right|^{2}}{\left(s^{2}+(\mu r)^{2}\right)^{3 / 2}} d s .
$$

Equation (3.28) indicates that $\Delta F_{1 \text {,ren }}^{l}$ can now be computed by first summing over the bound state energies, then the continuum state contributions can be evaluated by explicitly computing the exact phase shift only up to $\Lambda_{\mathrm{WKB}}$, beyond which the WKB method provides an analytical expression that accounts for contributions at higher energies. Note that while the WKB procedure entails an approximation, its accuracy can be made such that the difference between the exact and the WKB results is smaller than the desired precision. Finally, summation over $l$ yields

$$
\Delta F_{1, \text { ren }}=\sum_{l}(2 l+1) \Delta F_{1, \text { ren }}^{l}
$$

Since $\Delta F_{T}$ is not divergent, it can be computed exactly using eq. (3.14) and (3.16), or by replacing the exact phase shift $\delta_{l}$ above a certain energy scale by the approximate WKB phase shift $\delta_{l}^{\mathrm{WKB}}$ given by eq. (3.25). 


\section{Application and Discussion}

In ref.[7] these methods have been used to calculate the free energy of an instanton configuration which determines the decay rate in a first order phase transition. The computation of $\Delta F_{1}$ will be described first. It is found that the accuracy available on conventional computers prevents a precise determination of this quantity when it is straight-forwardly evaluated as in eq. (2.8) - that is, by doing the bare sum and subtracting the counterterm, without a decomposition into partial waves. When $\Delta F_{1}$ is computed exactly, by utilizing such a decomposition, very high numerical accuracy is still required because for each $l$ the bare sum and $F_{l}^{\mathrm{ct}}(\Lambda)$ must be evaluated at a large value of the cut-off $\Lambda$. But since both quantities diverge as a function of $\Lambda$, we find that convergence with reasonable accuracy is still difficult to attain. In contrast, evaluation of $\Delta F_{1 \text {,ren }}$ using the WKB-improved method consisting of eq. (3.28) and (3.32) converges rapidly for much lower values of the cut-off $\Lambda_{\mathrm{WKB}}$ and typically only the first fifty partial waves need to be summed; the parameters required for convergence are very much dependent on the nature $V^{\prime \prime}(\tilde{\phi}(r))$ and the values we have quoted come from the potentials we have examined.

The exact computation of $\Delta F_{T}$ can be performed by evaluating eq. (3.14) and (3.17), but at high temperatures it is found that several hundred partial waves must be summed to attain convergence. When the exact phase shift is replaced by the approximate WKB expression at high energies, there is a reduction in the computational burden and the same number of angular momentum channels must be summed. The improvement is not marked as it was for $\Delta F_{1 \text {,ren }}$ in part because $\Delta F_{T}$ is not renormalized. The results of these computations are summarized in Tables 4 and 5 of ref.[7].

In summary, we have elucidated a method for the exact evaluation the effective action to one-loop. The WKB extrapolation scheme was devised to reduce the computational effort. These methods enable an efficient calculation of the free energy associated with a phase transition, as detailed above. However, the applicability of this method is not limited to this example. Rather, it can be utilized in a broader variety of problems involving the non-perturbative evaluation of observables in a non-uniform background in quantum field theory ${ }^{[9]}$ as well as in classical systems ${ }^{[10]}$. It can also be generalized to encompass theories with fermions. ${ }^{[11]}$ 


\section{Appendix: One-loop Renormalization of the Scalar Field Theory}

This Appendix discusses the one-loop and renormalization of the scalar field theory described by eq. (2.1). The classical vacuum $\phi_{v}$ satisfies

$$
V^{\prime}\left(\phi_{v}\right)=0 \text { and } \mu^{2}=V^{\prime \prime}\left(\phi_{v}\right)>0 .
$$

At one-loop the only divergent graphs are those with one and two vertices corresponding to quadratic and logarithmic divergences, respectively.

It is convenient to adopt a renormalization scheme where the counterterms are chosen to exactly cancel the divergent graphs as shown in Figs. 1 and 2. These conditions are imposed at zero external momenta; this choice has the advantage that the one-loop contribution to the effective potential $V_{1}$ satisfies

$$
V_{1}\left(\phi_{v}\right)=V_{1}^{\prime}\left(\phi_{v}\right)=V_{1}^{\prime \prime}\left(\phi_{v}\right)=0,
$$

so that eq. (1) is unchanged at one-loop. Then the counterterm Lagrangian to be added to eq. $(2.1)$ is

$$
\mathcal{L}_{\mathrm{ct}}=\alpha\left[V^{\prime \prime}(\phi)-\mu^{2}\right]+\frac{1}{2} \beta\left[V^{\prime \prime}(\phi)-\mu^{2}\right]^{2},
$$

where

$$
\alpha=\frac{1}{2} \int \frac{i}{k^{2}-\mu^{2}+i \epsilon} \frac{d^{4} k}{(2 \pi)^{4}},
$$

and

$$
\beta=\frac{1}{2} \int \frac{i}{\left(k^{2}-\mu^{2}+i \epsilon\right)^{2}} \frac{d^{4} k}{(2 \pi)^{4}} .
$$

The terms in eq. (3) involving $\alpha$ and $\beta$ renormalize the graphs with one and two external vertices, respectively. These divergent integrals can be suitably regularized by imposing a momentum cut-off $\Lambda$.

\section{Acknowledgements}

The author wishes to thank David Wasson and David Brahm for enlightening discussions. 


\section{References:}

1. R. F. Dashen, B. Hasslacher \& A. Neveu, Phys. Rev. D10:4130 (1974).

2. D. A. Wasson, Ph.D. thesis, California Institute of Technology, 1990.

3. M. Li \& R. J. Perry, Phys. Rev. D37:1670 (1988).

4. J. Baacke, Z. Phys. C47:263 (1990).

5. J. Baacke \& V. G. Kiselev, DO-TH-93/18.

6. See for example, L.-H. Chan, Phys. Rev. Lett. 54:1222 (1985); Phys. Rev. Lett. 56:404(E) (1986).

7. D. E. Brahm \& C. L. Y. Lee, CALT-68-1881 (Nov. 1993).

8. D. E. Brahm \& C. L. Y. Lee, work in progress.

9. A detailed discussion can be found in R. Rajaraman, Solitons and Instantons, NorthHolland Press, Amsterdam, 1982).

10. For a survey, see Phase Transitions and Critical Phenomena, (Academic Press, London, 1983), Vol. 8, edited by C. Dom \& J. L. Lebowitz.

11. C. L. Y. Lee, work in progress. 


\section{Figure Captions}

Figure 1. Renormalization scheme for the divergent one-loop graphs with one vertex. A box with a cross denotes a counterterm insertion.

Figure 2. Renormalization scheme for the divergent graphs at one-loop order with two vertices. 